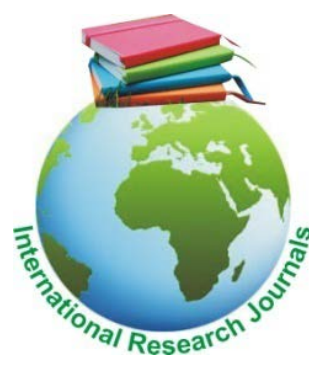

Journal of Research in Environmental Science and Toxicology Vol. 7(2) pp. 18-26, May, 2018

DOI: http:/dx.doi.org/10.14303/jrest.2018.016

Available online http://www.interesjournals.org/JREST

Copyright (C2018 International Research Journals

Full Length Review Paper

\title{
Impact of sulphate and heavy metals release on household portable water in Nigeria
}

\author{
Igibah C Ehizemhen*, Agashua Lucia O \\ Department of Civil Engineering, University of Abuja, F.C.T Abuja, Nigeria \\ E-mail: igibahchrist1@gmail.com
}

\begin{abstract}
The paper attempts frequent hitches regarding household portable water in Nigeria. It enlighten both end user and portable water providers about the heavy metals potential discharge that pre-accumulate in iron corrosion scales as it poses an contaminants health threat when released into household portable water. Likewise offers conjectural knowledge about formation, main uses, health effects of both heavy metals and sulphate on human for example arsenal causes immunotoxic, zinc creates abdominal pain etcetera besides common methods for removing both sulphate and heavy metals like distillation or reverse osmosis for small water and ion exchange for large water quantities because chemical reaction between sulfates and heavy metals discharge from portable water distribution system can affect the water quality by decreasing or oxidizing the heavy metals in the water therefore, awareness about the impacts of sulfate on heavy metals release from portable water distribution system is required . Also users and portable water provider must develop habit of testing portable water from consumer tap because despite water quality parameters of the finished water reached portable water healthy standards from water distribution system nonetheless the tap water which the customers exactly exploited could be infected, since the portable water undertake an extensive Hydraulic Retaining Period (HRP) of storing and transference in water distribution systems.
\end{abstract}

Key words: Sulphate, Heavy metals, household portable water, Nigeria

\section{INTRODUCTION}

Portable water quality ought to remain constant from the portable water treatment plant to the end user tap (Gunatilake, 2015). Even though all water quality parameters of the finished water reached portable water healthy standards from water distribution system nevertheless the tap water that the clienteles exactly exploited could be infested, because the portable water undertake a long Hydraulic Retaining Period (HRP) of storage and transference in water distribution systems (Manjie et al., 2016). Heavy metals can bond with sulfate compounds introduced into Portable Water Distribution Systems (PWDS) through chemicals utilized as coagulants in treating portable water. For instances iron and aluminium salts, like polyaluminium chloride, aluminium sulphate, as well ferric sulphate. Heavy metals accumulation in the Portable Water Distribution Systems (PWDS) has generated substantial concern, though presence of heavy metals is at concentrations beneath their Maximum Toxin Levels (MTL), they can gather within deposits besides corrosion scales can occur in PWDS (Huifang et al., 2017). Chemical reaction amongst sulphates and heavy metals released from portable water distribution system could affect the water quality by decreasing or rusting the heavy metals in the water. Sulphate was supposed to disturb the iron pipe oxidization as well result to red water in numerous cases, however the involved alleyway has not been well understood (Torres et al., 2015). Certain scholars hold the proposition that sulphate within iron oxidization tubercles intensification of the acidity within the tubercles, thus promoting metal corrosion besides corrosion products dissolution (Andrade et al., 2015). Decomposition scales disturb water quality inside Portable Water Distribution Systems (PWDSs) in many significant ways, like the degrees at which such scales are spawned, structures, chemical configuration, morphologies and solubility's of leading mineralogical levels instituting them as well make all affected through the pipe material for instance cast iron, PVC (Poly Vinyl Chloride) or steel on which they are deposited. Also water chemistry parameters that encompass of Dissolved Oxygen (DO), pH, alkalinity, 
temperature, chloride, sulphate concentrations, natural organic matter, disinfectant brand, residual, corrosion inhibitors presence like phosphate, water overall conductivity and hydraulic patterns (Hug et al., 2017; Ling et al., 2015). Furthermore, Sulphate Reducing Bacteria (SRB) have long been deliberated to be associated with the iron corrosion promotion as well hindering the stable iron oxide $\left(\mathrm{Fe}_{3} \mathrm{O}_{4}\right)$ formation, then substitute water springs plus long distance water transference could help in resolving water scarcity problems (Andrade et al., 2015). Since, source water alterations can cause adverse water quality changes because of the corrosion scales disturbance through changes in water chemistry (Agnieszka et al., 2018). Sulphide that was transformed into sulphate creating a black slime that can clog plumbing as well stain clothing, water blackening or black slime coating within toilet tanks which might be revealing a sulfur-oxidizing bacteria problem. Sulphate can initiate heavy metal discharge from iron decomposition scales since there are strong correlations incidence amongst the heavy metals releases or toxic metals which are the metal compounds that are toxic besides have negative influence on publics' health but very slight quantities of these metals are mandatory to support life (Ghaderpoori et al., 2018; Hug et al., 2017). In recent years, several serious red water cases have occurred due to source water swap, for instance in Southern California, Tucson, Arizona and Tampa, Florida in the United States (Liu et al., 2017). In October, 2008 red water appeared in some regions in a city in Northern China soon after eighty percent (8\%) of their indigenous source water was altered with source water from a neighboring area. It was seeming that the zones suffering from red water were historically supplied with local groundwater, but the regions without red water were historically provided with local surface waters and the new water source had considerably higher sulfate equated with the local groundwater roughly 200 vns. $30 \mathrm{mg} / \mathrm{L}$. The Tucson, Arizona and Tampa, Florida red water problems that were elicited through source water modifications from groundwater to surface water, these existences indicated that the formation and corrosion scales morphology played substantial roles in changes of distribution water quality from the corrosion scales alliance with finished water. Furthermore, some scholars hold the hypothesis that sulfate within iron corrosion tubercles intensification the acidity inside the tubercles, thus promoting metal corrosion and suspension of the corrosion products (Ghaderpoori et al., 2018; Hug et al., 2014).

\section{LITERATURE REVIEW}

\section{Sulphate and Heavy Metal Formation}

Soil and rock creations might naturally contain sulphates and as water moves through these, sulphates are picked up besides liquefy in the groundwater (Hug et al., 2014). Sulphates transpire as minuscular particles (vaporizers) ensuing from fossil fuel plus biomass ignition, they increase the atmosphere acidity and generate acid rain (Brraich and Jangu, 2015). Several sulphates were known to pseudoscientists for examples Alum, a twice potassium and aluminium sulphate with the formula $\mathrm{K}_{2} \mathrm{Al}_{2}\left(\mathrm{SO}_{4}\right)_{4} \cdot 24 \mathrm{H}_{2} \mathrm{O}$ which figured in the chemical industry improvement and the vitriol salts, the first translucent crystals recognized; blue vitriol which is copper (II) sulphate pentahydrate $\left(\mathrm{CuSO}_{4} \cdot 5 \mathrm{H}_{2} \mathrm{O}\right)$; green vitriol is iron (II) sulphate heptahydrate, $\mathrm{FeSO}_{4} \cdot 7 \mathrm{H}_{2} \mathrm{O}$ and white vitriol is zinc sulfate heptahydrate, $\mathrm{ZnSO}_{4} \cdot 7 \mathrm{H}_{2} \mathrm{O}$ (Watts et al., 2017; Balakrishan and Ramu, 2016; Ediagbonya et al., 2015). The anaerobic Sulphate Reducing Bacteria (SRB)

Desulfovibrio vulgaris and $D$. desulfuricans eradicate the black sulphate layer that frequently taints buildings besides SRB removed metals by the production of highly insoluble precipitates with biogenic sulfide as shown below:

$\mathrm{H}_{2} \mathrm{~S}+\mathrm{M}^{2+} \mathrm{a}+\mathrm{MS}(\mathrm{s})+2 \mathrm{H}+(2)$

where $\mathrm{M}^{2+}=$ metal, such as $\mathrm{Zn}^{2}+, \mathrm{Cu}^{2}+, \mathrm{Pb}^{2}+$ and $\mathrm{Ni}^{2+}$

SRB are either use organic compounds (heterotrophic) or by the means of hydrogen as an electron electron donor and $\mathrm{CO}_{2}$ as carbon source (autotrophic) anaerobes, able of decreasing sulfate to sulfide by a dissimilatory, bioenergetic digestion.

Acid Mine Drainage (AMD) emanations from mining actions generate pollution difficulties into the environment, when expose to water, oxidizing bacteria presence, oxygen, pyrite and other sulphide minerals they dissolved and produce a leachate comprising sulphate, dissolved metals and acidity as shown in Figure 1.

Heavy metal denotes any type of metal plus metalloid component with relatively great density varying from three point five (3.5) to seven (7) $\mathrm{gcm}^{-3}$, they are noxious as well

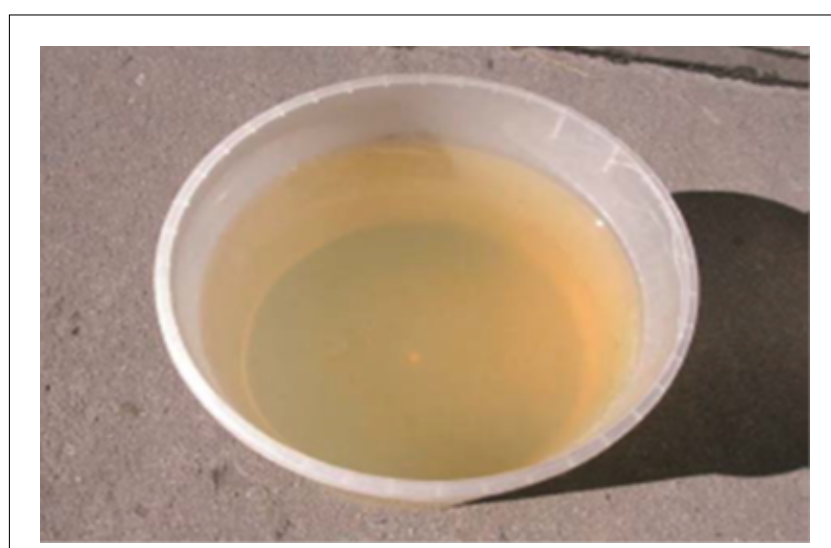

Figure 1: Stained tap water sourced in an apartment 
Table 1: Sulphate molecular formula name

\begin{tabular}{|c|c|l|}
\hline $\mathbf{S} / \mathbf{N}$ & Sulphate Names & Formula \\
\hline $\mathbf{1}$ & Peroxomonosulphate & $\mathrm{SO}^{2-}{ }_{5}$ \\
\hline $\mathbf{2}$ & Sulphate & $\mathrm{SO}^{2-}{ }_{4}$ \\
\hline $\mathbf{3}$ & Sulphite & $\mathrm{SO}^{2-}{ }_{3}$ \\
\hline $\mathbf{4}$ & Peroxydisulphate & $\mathrm{S}_{2} \mathrm{O}^{2-}{ }_{8}$ \\
\hline $\mathbf{5}$ & Pyrosulphate & $\mathrm{S}_{2} \mathrm{O}^{2-}{ }_{7}$ \\
\hline $\mathbf{8}$ & Dithionate & $\mathrm{S}_{2} \mathrm{O}^{2-}$ \\
\hline $\mathbf{9}$ & Metabisulphite & $\mathrm{S}_{2} \mathrm{O}^{2-}{ }_{5}$ \\
\hline $\mathbf{1 0}$ & Dithionite & $\mathrm{S}_{2} \mathrm{O}^{2-}{ }_{4}$ \\
\hline $\mathbf{1 1}$ & Thiosulphate & $\mathrm{S}_{2} \mathrm{O}^{2-}{ }_{3}$ \\
\hline $\mathbf{1 2}$ & Trithionate & $\mathrm{S}_{3} \mathrm{O}^{2-}{ }_{6}$ \\
\hline $\mathbf{1 3}$ & Tetrathionate & $\mathrm{S}_{4} \mathrm{O}^{2-}{ }_{6}$ \\
\hline
\end{tabular}

deadly even at low concentrations as shown in Table 1. Though heavy metals is a universal term that is extensively documented besides often applied to the extensive soils and water bodies contaminants (Fish et al., 2017; Ghaderpoori et al., 2018). They are found broadly in the earth's layer and are non-decomposable in nature, they might enter into the environment or nearby water bodies by household applications, mining events and industrial discharge. They likewise enter into the people body through food, air and water although a slight quantity has vital role in the humans and animals digestion however their higher concentration could cause noxiousness and health hazards (Moreau, 2017). According to WHO, pipeline internal corrosion can augment heavy metals like $\mathrm{Pb}, \mathrm{Cu}$ and $\mathrm{Fe}$ into portable water, besides, iron will cause detrimental taste and colour to the delivered portable water (Andrade et al., 2015; Gunatilake, 2015) $\mathrm{SO}_{2}^{-5}$ as shown in Figures $2 a$ and $b$.

\section{Main Uses of Sulphate and Heavy Metals}

Sulphate salts have various applications for instances; gypsum is the natural mineral kind of hydrated calcium sulphate and is utilized in plaster making, nearly one hundred million tonnes annually are consumed by the construction company; copper sulphate, well-known algaecide, its more stable form $\mathrm{CuSO}_{4}$ is utilized for galvanic cells as electrolyte and for fungicide that controls bacterial and fungal diseases of crops. Iron (II) sulphate, iron common form in mineral complements is used for soil plants, humans and animals; Lead (II) sulphate, generated on both plates throughout lead-acid battery discharge; magnesium sulfate, or epsom salt, is used for therapeutic baths. Other sulphates comprise of glucosamine sulphate, used for treating arthritis and hydrazine sulphate, commonly used in treating cancer patients. Heavy metals examples are: $\mathrm{Pb}$ (lead), $\mathrm{Hg}$ (mercury), $\mathrm{Cr}$ (chromium), Cd (cadmium), Ni (nickel), As (arsenic), $\mathrm{Cu}$ (copper), $\mathrm{Ti}$ (thallium) and $\mathrm{Zn}$ (zinc) as shown in Tables 2 and 3.

Cadmium is widely utilized in electroplating companies, batteries, welds, television sets, ceramic ware,

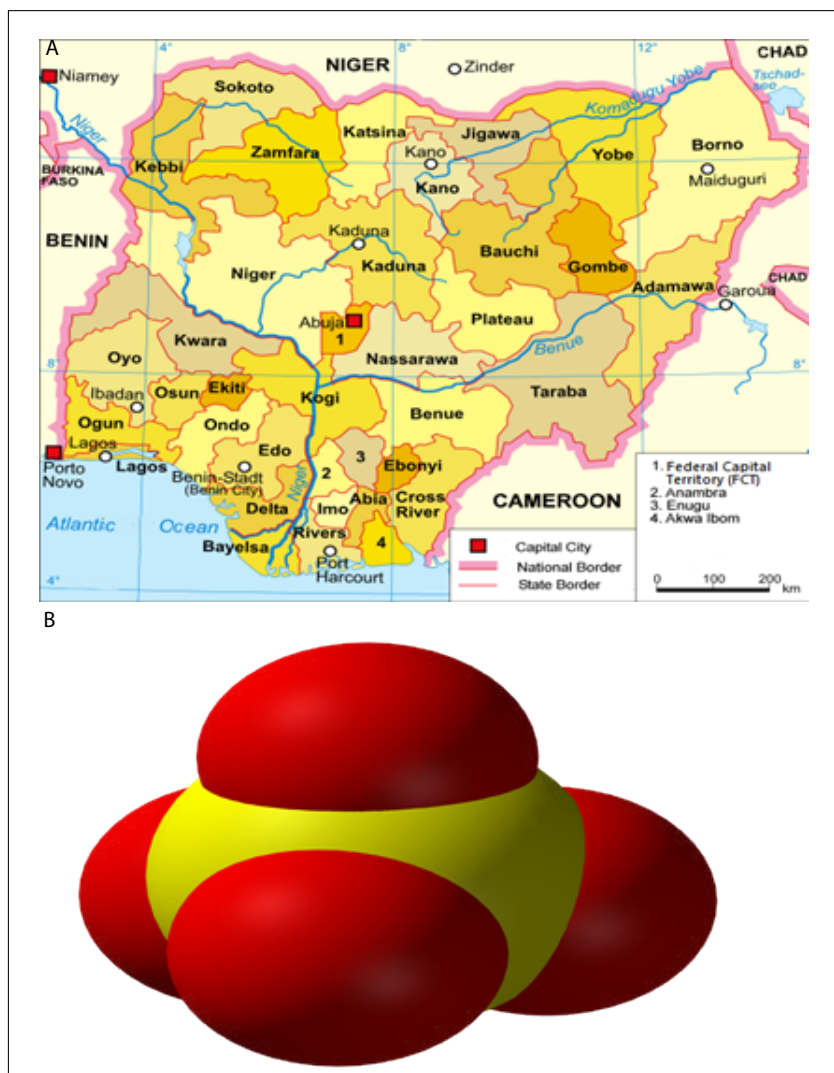

Figure 2: (a) Map of Nigeria; (b) Ball-and-stick model of the sulfate anion

photography, pesticides, electronic engineering, metalfinishing companies and metallurgical events. Copper, an indispensable element which is obligatory through biological systems for some enzymes instigation lower concentrations throughout photosynthesis. Nickel and its salts are utilized in numerous industrial uses like coating, coins, automobile, parts of aircraft, batteries, catalyst plugs, make-ups, stainless steel, besides is utilized comprehensively in the nickel-cadmium batteries manufacture on an industrial scale. Zinc are utilized in the production of galvanized iron, veneers, bronze, rubber, enamel glass, fertilizers, paper, as a wood protective $\left(\mathrm{ZnCl}_{2}\right.$, fungicidal action), white paint, petrochemicals and in steam generation power plants. Mercury is obtained from metal broad usage in manufacturing applications, its mining, processing besides batteries uses and mercury gas lamps. Chromium is from the chromates making, fossil energies burning, plastic production, metals coating and broad usage in the tannery plus leather industries. Cobalt compounds are utilized as pigments in paints, glass and ceramics, besides as paint driers, catalysts, trace element extracts in agriculture and medicine.

\section{Effects of Sulphate and Heavy Metals on Human}

Sulphate high levels might lead to temporary desiccation, 
Table 2: Heavy metals, their salts and other compounds chart

\begin{tabular}{|c|c|c|c|}
\hline $\begin{array}{l}\text { Physical description } \\
\text { Chemical formular }\end{array}$ & Mineral & Uses & $\begin{array}{l}\text { Exposure } \\
\text { Level }\end{array}$ \\
\hline $\begin{array}{l}\text { Aluminum sulfate } \mathrm{Al}_{2}\left(\mathrm{SO}_{4}\right)^{3} \\
\text { Odorless white rock crystal or powder. Alum } \\
\text { compounds is Aluminum sulphate (aluminum } \\
\text { sulfate, water, and others e.g. potassium, } \\
\text { sodium, ammonium). Potassium aluminum } \\
\text { sulfate } \\
\mathrm{K}_{2} \mathrm{SO}_{4} \cdot \mathrm{Al}_{2}\left(\mathrm{SO}_{4}\right)^{3} \cdot 24 \mathrm{H}_{2} \mathrm{O} \\
\text { White powder or rock crystal } \\
\text { Aluminum ammonium sulfate } \\
\text { AlNH } \mathrm{SO}_{4}\left(\mathrm{SO}_{4}\right)^{2} \cdot 12 \mathrm{H}_{2} \mathrm{O} \\
\text { White crystal-like powder }\end{array}$ & $\begin{array}{l}\text { Aluminum (sulphate, trisulphate, (III) } \\
\text { sulfate, Alum kinds e.g (filter, meal, } \\
\text { cake, cube, stone, flour, patent, paper } \\
\text { makers, pearl, pickle, sulfuric acid, } \\
\text { native, potash, alumina, alumnus; } \\
\text { ammonia and alumnae) }\end{array}$ & $\begin{array}{l}\text { human immunizations, } \\
\text { antibacterial agent, antiseptic, } \\
\text { emetic, purification of water, } \\
\text { hair stiffener, treatments of } \\
\text { sewage, fire retardant, food } \\
\text { conserving, food seasoning, } \\
\text { cement, textile dying, paints; } \\
\text { white photography; } \\
\text { baking powder and leather } \\
\text { tanning }\end{array}$ & $\begin{array}{l}\text { NIOSH REL, TBV: two } \\
\mathrm{mg} / \mathrm{m}^{3} \text { as Al soluble } \\
\text { salts } \\
\text { ACGIH 1993- } \\
1994\end{array}$ \\
\hline $\begin{array}{l}\text { Antimony sulphide } \mathrm{Sb}_{2} \mathrm{~S}_{3} \\
\text { Odorless orange plus red to black crystal-like } \\
\text { solid. }\end{array}$ & $\begin{array}{l}\text { Antimony sulphide, antimony } \\
\text { sulphide, Antimonial glass, chloride } \\
\text { plus sodium thiosulphate or hydrogen } \\
\text { sulphide. }\end{array}$ & $\begin{array}{l}\text { Rubber company pigments, } \\
\text { ruby glass colorant, facade } \\
\text { paints. }\end{array}$ & $\begin{array}{l}\text { OSHAAES: PWA } \\
0.5 \mathrm{mg} / \mathrm{m}^{3}\end{array}$ \\
\hline $\begin{array}{l}\text { Antimony trisulphide } \mathrm{Sb}_{2} \mathrm{~S}_{3} \\
\text { Red-orange crystalline solid. }\end{array}$ & $\begin{array}{l}\text { Antimony kinds e.g (Orange, red, } \\
\text { sulphide and (III) sulphide). }\end{array}$ & $\begin{array}{l}\text { ruby glass colorant, fireworks } \\
\text { and matches. }\end{array}$ & $\begin{array}{l}\text { OSHA AES: PWA } \\
0.5 \mathrm{mg} / \mathrm{m}^{3} \text {. }\end{array}$ \\
\hline $\begin{array}{l}\text { Arsenic sulphide } \mathrm{AsS} / \mathrm{As}_{2} \mathrm{~S}_{2} / \mathrm{As}_{2} \mathrm{~S}_{4} \\
\text { Lustrous to translucent orange-red to yellow } \\
\text { orange crystals. }\end{array}$ & $\begin{array}{l}\text { Arsenic kinds e.g (orange, red, } \\
\text { sulphide, disulphide Realgar, red } \\
\text { orpiment and ruby sulphur). }\end{array}$ & $\begin{array}{l}\text { Fireworks, paper, leather } \\
\text { tanning, textiles and colorants } \\
\text { geological. }\end{array}$ & $\begin{array}{l}\text { OSHAAES: } \\
1910.1018 \\
\text { PWA } 0.01 \mathrm{mg} / \mathrm{m}^{3}\end{array}$ \\
\hline $\begin{array}{l}\text { Arsenic trisulphide } \mathrm{As}_{2} \mathrm{~S}_{3} \\
\text { Orange-yellow to lemon yellow crystals }\end{array}$ & $\begin{array}{l}\text { Arsenic kinds e.g (sulphide, (III) } \\
\text { sulphide and yellow). }\end{array}$ & $\begin{array}{l}\text { glass Fireworks, paper and } \\
\text { x-ray transmitting assembly }\end{array}$ & $\begin{array}{l}{[1910.1018] \text { PWA }} \\
0.01 \mathrm{mg} / \mathrm{m}^{3}\end{array}$ \\
\hline $\begin{array}{l}\text { Barium sulphate } \mathrm{BaSO}_{4} \text { Odorless, } \\
\text { yellowish or white rock crystal }\end{array}$ & $\begin{array}{l}\text { Barium sulphate; Artificial barite, } \\
\text { Barytes, Heavy spar, Permalba and } \\
\text { Bologna white. }\end{array}$ & $\begin{array}{l}\text { Pigments, Petroleum } \\
\text { manufacture, rubber and x-ray } \\
\text { imaging radiocontrast. }\end{array}$ & $\begin{array}{l}\text { OSHA AES: PWA } \\
0.5 \mathrm{mg} / \mathrm{m}^{3}\end{array}$ \\
\hline $\begin{array}{l}\text { Barium sulphide BaS } \\
\text { Pale grey powder }\end{array}$ & $\begin{array}{l}\text { Barium sulphide, Lapis solis, Black } \\
\text { ash, Barium monosulphide; }\end{array}$ & $\begin{array}{l}\text { Vulcanized rubber; de-hairing } \\
\text { hides; luminous paints pigment }\end{array}$ & $\begin{array}{l}\text { OSHA AES: PWA } \\
0.5 \mathrm{mg} / \mathrm{m}^{3}\end{array}$ \\
\hline $\begin{array}{l}\text { Barium thiosulphate } \mathrm{BaS}_{2} \mathrm{O}_{3} \cdot \mathrm{H}_{2} \mathrm{O} \\
\text { White crystal-like powder }\end{array}$ & $\begin{array}{l}\text { Barium (hyposulphite and } \\
\text { thiosulphate). }\end{array}$ & $\begin{array}{l}\text { Textile coloring, pigments and } \\
\text { graphic solutions. }\end{array}$ & $\begin{array}{l}\text { OSHA AES: PWA } \\
0.5 \mathrm{mg} / \mathrm{m}^{3}\end{array}$ \\
\hline $\begin{array}{l}\text { Cadmium sulphate } \mathrm{CdSO}_{4} \\
\text { Odorless white solid }\end{array}$ & $\begin{array}{l}\text { Cadmium sulphate; CragTurf } \\
\text { Fungicide; Cad-Trete. }\end{array}$ & $\begin{array}{l}\text { Fungicides for turf and tree } \\
\text { bark }\end{array}$ & $\begin{array}{l}0.01 \mathrm{mg} / \mathrm{m}^{3}, \mathrm{~A} 2 \\
(\mathrm{ACGIH} \mathrm{1998)}\end{array}$ \\
\hline $\begin{array}{l}\text { Cadmium sulphide CdS. Orange, light } \\
\text { yellow rock crystal or yellow to brown } \\
\text { powder. }\end{array}$ & $\begin{array}{l}\text { Cadmium (yellow, monosulphide, } \\
\text { lemon yellow, orange and yellow } \\
\text { conc.). }\end{array}$ & $\begin{array}{l}\text { Coatings on photovoltaic cells } \\
\text { and pigments }\end{array}$ & $\begin{array}{l}\text { TBV: } 0.01 \mathrm{mg} / \mathrm{m}^{3} \\
\text { A2, ACGIH } 1996\end{array}$ \\
\hline $\begin{array}{l}\text { Calcium sulphate } \mathrm{CaSO}_{4} \text {. Colorless rock } \\
\text { crystal or unscented white powder, can be } \\
\text { gray tinge, reddish or blue. } \\
\text { Calcium sulphate dehydrate } \mathrm{CaSO}_{4} \\
2 \mathrm{H}_{2} \mathrm{O} \text {. Unscented colorless or white powder, } \\
\text { reddish tinge, gray or blue. }\end{array}$ & $\begin{array}{l}\text { Calcium sulphate (Blue marble, } \\
\text { gypsum i.e plaster of Paris (POP) and } \\
\text { anhydrous lime sulphate. }\end{array}$ & $\begin{array}{l}\text { Ceramics composition and fills, } \\
\text { plaster, polychrome sculpture } \\
\text { filler, paper filler, plastics, dyes } \\
\text { and chalkboard }\end{array}$ & $\begin{array}{l}\text { OSHA AES: PWA } \\
15 \mathrm{mg} / \mathrm{m}^{3} \text { total; } 5.0 \\
\mathrm{mg} / \mathrm{m}^{3}\end{array}$ \\
\hline $\begin{array}{l}\text { Chromic sulphate } \mathrm{Cr}_{2}\left(\mathrm{SO}_{4}\right)^{3} \text {. Numerous } \\
\text { hydrated forms, green and violet-red powder }\end{array}$ & Chromium sulphate & $\begin{array}{l}\text { Leather tanning, ceramic } \\
\text { glazes, printing textiles, } \\
\text { varnishes and inks. }\end{array}$ & $\begin{array}{l}\text { OSHA AES: PWA } \\
0.5 \mathrm{mg} / \mathrm{m}^{3}\end{array}$ \\
\hline $\begin{array}{l}\text { Chromium potassium sulphate } \\
\mathrm{CrK}\left(\mathrm{SO}_{4}\right) 2 \cdot 12 \mathrm{H}_{2} \mathrm{O} \text { Dark violet crystals }\end{array}$ & $\begin{array}{l}\text { Chrome (alum, potash alum and } \\
\text { potassium alum) }\end{array}$ & $\begin{array}{l}\text { Photographic processing } \\
\text { solutions hardener and leather } \\
\text { tanning. }\end{array}$ & $\begin{array}{l}\text { OSHAAES: PWA } \\
0.5 \mathrm{mg} / \mathrm{m}^{3}\end{array}$ \\
\hline $\begin{array}{l}\text { Cobaltous ammonium sulphate } \\
\mathrm{CoSO}_{4^{-}}\left(\mathrm{NH}_{4} \mathrm{SO}_{4}^{-}-6 \mathrm{H}_{2} \mathrm{O}\right. \\
\text { Ruby red rock crystal }\end{array}$ & & $\begin{array}{l}\text { ceramic veneers, cobalt plating } \\
\text { and enamels colorant. }\end{array}$ & $\begin{array}{l}\text { TBV: } 0.02 \mathrm{mg} / \mathrm{m}^{3} \text { as } \\
\text { PWA, A3 ACGIH } 1997\end{array}$ \\
\hline $\begin{array}{l}\text { Copper sulphate (anhydrous) } \mathrm{CuSO}_{4} \text { pale } \\
\text { green or white hygroscopic rock crystal. }\end{array}$ & $\begin{array}{l}\text { Sulfuric acid, Blue stone, Copper (II) } \\
\text { salts and sulfate. }\end{array}$ & $\begin{array}{l}\text { Crystal grow, hair dyes, glass } \\
\text { colorant, herbicides and } \\
\text { pesticides. }\end{array}$ & $\begin{array}{l}\text { OSHA AES: PWA } \\
1.0 \mathrm{mg} / \mathrm{m}^{3} \text { copper } \\
\text { dusts \& mists }\end{array}$ \\
\hline $\begin{array}{l}\text { Ferric ammonium sulphate } \mathrm{FeNH}_{4} \\
\left(\mathrm{SO}_{4}\right) 2 \cdot 12 \mathrm{H}_{2} \mathrm{O} \text {. Pale lilac rock crystal; air } \\
\text { effloresce and light green crystal-like solid. }\end{array}$ & $\begin{array}{l}\text { Ferric (alum, ferrous sulfate, } \\
\text { ammonium iron sulfate or Mohr's } \\
\text { Salt). }\end{array}$ & $\begin{array}{l}\text { Medicine, textile dying caustic, } \\
\text { toning answers for black \& } \\
\text { white photography. }\end{array}$ & $\begin{array}{l}\text { TLV: } 1.0 \mathrm{mg} / \mathrm{m}^{3} \mathrm{TWA} \\
\text { as soluble iron salt, } \\
\text { ACGIH) }\end{array}$ \\
\hline $\begin{array}{l}\text { Lead sulphide PbS. Black to silvery powder } \\
\text { or crystal-like solid. }\end{array}$ & $\begin{array}{l}\text { Galena (lead main source) and } \\
\text { Plumbous sulphide. }\end{array}$ & $\begin{array}{l}\text { Feudal pottery, photon } \\
\text { detectors; ceramic glazes and } \\
\text { geological samplings. }\end{array}$ & $\begin{array}{l}\text { OSHA AES: } 1910 . \\
\text { 1025, PWA } 0.05 \mathrm{mg} / \\
\mathrm{m}^{3}\end{array}$ \\
\hline $\begin{array}{l}\text { Magnesium sulphate } \mathrm{MgSO}_{4} \\
\text { White crystals or powder }\end{array}$ & $\begin{array}{l}\text { Magnesium sulphate or Epsom salts } \\
\text { or Epsomite }\end{array}$ & $\begin{array}{l}\text { Plants soil additive, bath salts } \\
\text { and water additive. }\end{array}$ & TBV not established \\
\hline
\end{tabular}




\begin{tabular}{|c|c|c|c|}
\hline $\begin{array}{l}\text { Mercuric sulphate } \mathrm{HgSO}_{4} \\
\text { Unscented, white crystal-like powder } \\
\text { Mercuric sulphide } \mathrm{HgS} \\
\text { Unscented red-orange or dark powder }\end{array}$ & $\begin{array}{l}\text { Mercury (red, bisulphate, black, (II) } \\
\text { sulphate, sulphide; (II) sulphide and } \\
\text { Cinnabar). }\end{array}$ & $\begin{array}{l}\text { Gold and silver mining, } \\
\text { medicine, Dyes, stamps, } \\
\text { geological samplings }\end{array}$ & $\begin{array}{l}\text { Ppm } 0.025 \mathrm{mg} / \mathrm{m} 3 \\
\text { OSHAAES: } 0.1 \mathrm{mg} / \\
\mathrm{m}^{3}, \text { TBV: } 0.05 \mathrm{mg} / \mathrm{m} 3\end{array}$ \\
\hline $\begin{array}{l}\text { Zinc sulphate heptahydrate } \mathrm{ZnSO}_{4} \cdot 7 \mathrm{H}_{2} \mathrm{O} \text {. } \\
\text { Granules or crystal-like powder }\end{array}$ & Goslarite \& white vitriol & $\begin{array}{l}\text { skin \& leather additive, } \\
\text { medicine \& textile acerbic. }\end{array}$ & TBV not established \\
\hline
\end{tabular}

Table 3: Portable water standard metal concentration and the health impacts

\begin{tabular}{|c|c|c|c|}
\hline S/N & Metals & Impacts & Portable water Standard \\
\hline 1 & Lead & $\begin{array}{ll}\text { - High doses generate metabolic poison } \\
\text { - } \\
\text { batigue, irritability anemia and children } \\
\text { behavioral changes. } \\
\text { Hypertension and brain damage. }\end{array}$ & $\begin{array}{l}\text { - Using EPA maximum concentration: } 0.1 \mathrm{mgL}^{-1} \\
\text { EC: } 0.5 \mathrm{mg} \mathrm{L}^{-1} \text {. }\end{array}$ \\
\hline 2 & Nickel & $\begin{array}{ll} & \text { High conc. could generate DNA damage } \\
\text { - } & \text { Hands eczema } \\
\text { High phytotoxicity }\end{array}$ & $\begin{array}{l}\text { - Using EPA maximum concentration: } 0.1 \mathrm{mgL}^{-1} \\
\text { - } \quad \text { EC: } 0.1 \mathrm{mgL}^{-1}\end{array}$ \\
\hline 3 & Chronium & $\begin{array}{ll}\text { - } & \text { Gastrointestinal mucosa irritation } \\
\text { - } & \text { Necrosis nephritis besides death in man } \\
\end{array}$ & $\begin{array}{ll}\text { - } & \text { Using EPA maximum concentration: } 0.1 \mathrm{mgL}^{-1} \\
\text { - } & \text { EC: } 0.5 \mathrm{mgL}^{-1}\end{array}$ \\
\hline 4 & Copper & $\begin{array}{ll}\text { - } & \text { Creates injury in aquatic fauna variety. } \\
\text { - } & \text { Corrosion and mucosal irritation } \\
\end{array}$ & $\begin{array}{l}\text { - Using EPA maximum concentration: } 0.1 \mathrm{mgL}^{-1} \text {. } \\
\text { - } \\
\text { EC: } 0.1 \mathrm{mgL}^{-1}\end{array}$ \\
\hline 5 & Cadmium & $\begin{array}{l}\text { - Generates serious kidneys and bones injury in } \\
\text { humans. }\end{array}$ & $\begin{array}{l}\text { - Using EPA maximum concentration: } 0.005 \mathrm{mgL}^{-1} \\
\text { - }\end{array}$ \\
\hline 6 & Arsenic & $\begin{array}{l}\text { - } \quad \text { Creates toxicological plus cancer-causing } \\
\text { effects. } \\
\text { - Immunotoxic }\end{array}$ & $\begin{array}{l}\text { - WHO guideline: } 10 \mu \mathrm{gL}^{-1} . \\
\text { - }\end{array}$ \\
\hline 7 & Zinc & $\begin{array}{ll}\cdot & \text { Phytotoxic } \\
\cdot & \text { Anemia } \\
\text { - } & \text { Abdominal Pain }\end{array}$ & $\begin{array}{l}\text { - using Environmental protection Agency maximum } \\
\text { concentration: } 5 \mathrm{mgL}^{-1} \\
\text { - } \quad \text { European community: } 5 \mathrm{mgL}^{-1}\end{array}$ \\
\hline 8 & Mercury & $\begin{array}{ll}\text { - } & \text { Toxic } \\
\text { - } & \text { Generates mutagenic effects } \\
\text { - } & \text { Disrupts the cholesterol }\end{array}$ & $\begin{array}{l}\text { - Using Environmental protection Agency maximum } \\
\text { concentration: } 0.002 \mathrm{mgL}^{-1} \\
\text { - } \quad \text { European community: } 0.001 \mathrm{mgL}^{-1}\end{array}$ \\
\hline
\end{tabular}

laxative effects when united with calcium plus magnesium that are the most popular features of hardness and copper pipes corrosion. Also bacteria which attack and decreases sulphates form hydrogen sulphides gas $\left(\mathrm{H}_{2} \mathrm{~S}\right)$, they are irritating as well dry out hair and skin. Copper highly toxic to various water organisms even at very small absorptions (Ediagbonya et al., 2015). Lead is terrifically noxious as well demonstrations deadliness to the reproductive organ, nervous system and kidneys. Lead exposure creates permanent brain damage and encephalopathic signs. Mercury toxicity has been acknowledged worldwide, for illustration in Japan Bay, Minamata, emotionally distressed and physically mutilated babes were born by unprotected mothers who consumed contaminated fish from toxic mercury.

\section{COMMON METHODS TO REMOVE SULPHATE AND HEAVY METALS FROM PORTABLE WATER}

Numerous techniques of eradicating sulphate from water are obtainable, the treatment scheme to be chosen hinge on many factors which include water sulphate level, iron quantity, manganese amount in the water plus bacteria contagion if available must also be treated. For treating small measures of portable water the typical approaches might be distillation or reverse osmosis whereas the most popular technique for treating big water quantities is ion exchange. Iron oxidation depend on the carbonate concentration, solution's $\mathrm{pH}$ as well organic matter. Corrosion of iron plus manganese could be attained through oxidizing agent introduction and this can be achieved by the oxidants addition like potassium and chlorine permanganate as shown in Figures $3 a$ and $b$.

\section{EPA Impact on Sulphate and its Compound}

The EPA (Environmental Protection Agency) portable water standards fall into two kinds; Primary and Secondary Standards. Primary Standards are centered on health concerns and are aimed at protecting populaces from three classes of noxious toxins viz; poisonous chemicals, pathogens and radioactive elements. Secondarystandards centered on corrosivity, taste, foaming, odor, water staining properties and color. Meanwhile, sulfate is grouped under the Secondary Maximum Toxin Level (SMTL) standards and for value portable water is $250 \mathrm{mg} / \mathrm{L}$ or $250 \mathrm{ppm}$. Even though various impurities are controlled by Primary or Secondary Drinking Water Standards set by the EPA, hydrogen sulfide is not controlled because a concentration high sufficient to be a drinking water health hazard also makes the water unpleasant. The odor of water with as miniature as $0.5 \mathrm{ppm}$ of hydrogen sulfide concentration is noticeable by most people. Concentrations less than 1 ppm give the water a "fusty" or "moist" odor. A 1-2 ppm hydrogen sulfide concentration offers water a "rotten egg" odor and makes the water very caustic to plumbing. 


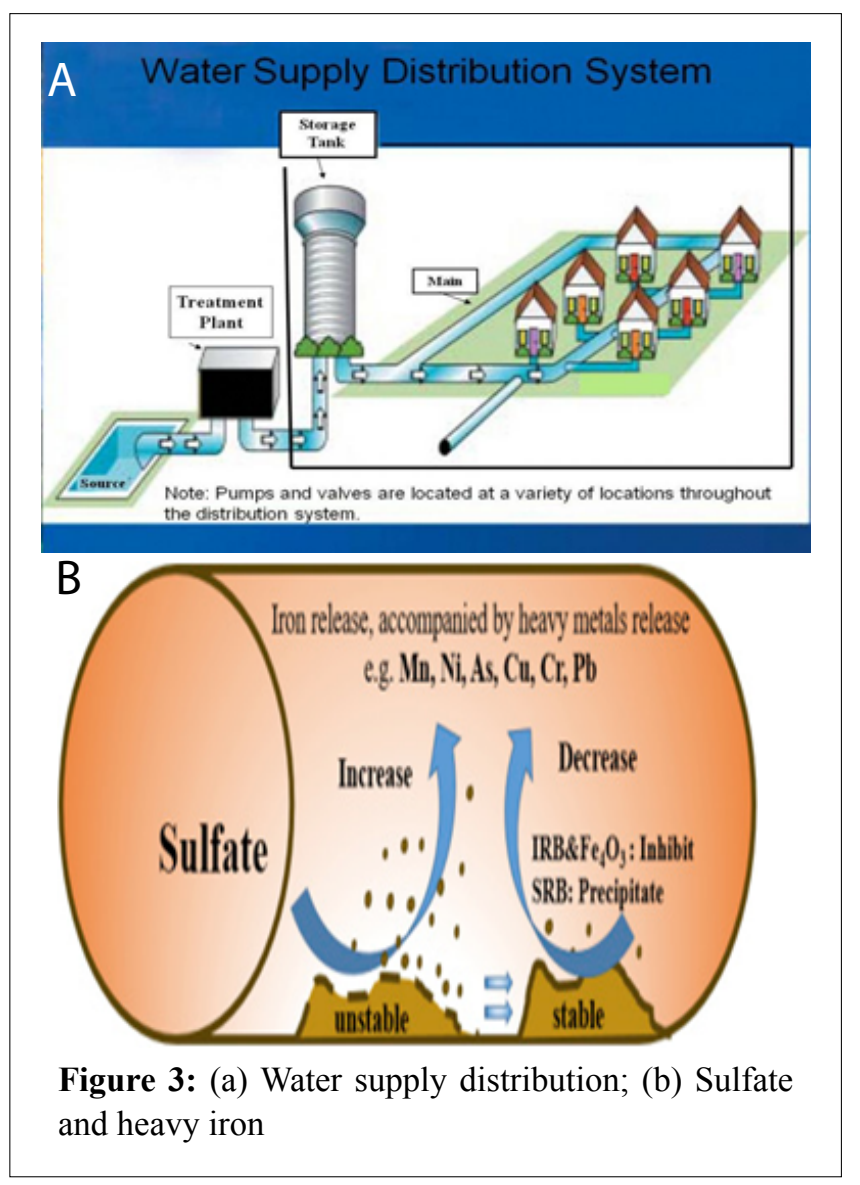

\section{Portable Water Supply Network}

This is a method of contrived hydrologic as well hydraulic mechanisms that deliver water supply and typically comprises of: Drainage basin; Natural water gathering point beyond or beneath ground where water accrues, like river, lake or groundwater from an underground aquifer besides this might be conveyed using covered tunnels, exposed ground-level conduits or underground water pipelines; Water filtering amenities: treated water is transported using water pipes that is frequently underground; Water storage amenities for instances water cisterns, reservoirs or water masts, lesser water methods can stockpile the water in reservoirs or pressure containers, high buildings will require storing water locally in pressure basins so that water can reach the higher floors. Supplementary water pressuring features for example pumping stations can be location at the underground vent, beyond ground reservoirs or reservoir if gravity flow is unachievable; Pipe setup for water distribution to the users which can be commercial, personal houses, industrial, institution establishments or other usage places like fire hydrants and underground pipes connections for sewers or above ground waterways in various unindustrialized nations are largely discovered at water consumers downstream, then the sewer method is deliberated to be dispersed system,

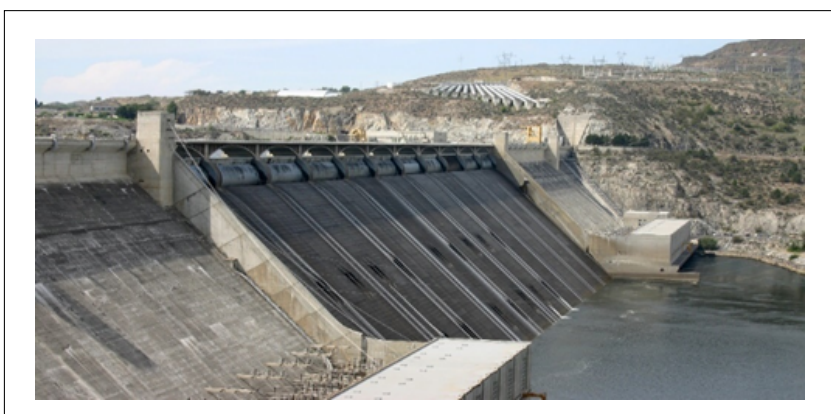

Figure 4: Grand Coulee Dam

instead of water supply system (Andrade et al., 2015) as shown in Figure 4.

\section{Necessities of Good Distribution System}

Distribution system purpose is to convey water to consumer with apposite quality, quantity and pressure besides is used to describe collectively the facilities tilized to supply water from its source to the usage point. Thus a good distribution system water quality must not be depreciated inside the distribution pipes, it must be competent in water distribution at all the projected places with appropriate pressure head besides the plan must be such that no user could be deprived of water supply even during any system sectional repair. All the distribution pipes ought to be positioned one metre $(1 \mathrm{~m})$ away or beyond the sewer lines and must be fairly water-tight to retain losses caused by leakage to tiniest.

\section{Option for Excess Sulphate Plus Heavy Metals in Portable Water}

In case of present of excessive sulfate in the water supply, there are three simple choices: First, utilize some kind of treatment to remove the contamination, based on the water chemical analysis outcomes through a reliable laboratory; secondly, acquire bottled water from stores or directly from bottling firms, this substitute can be considered specially when the major concern is water for food preparation and drinking; thirdly, whole-house treatment system installation which is the best cost effective system besides a function of the complete water quality, treat sulphate odor and other water treatment matters as shown in Figure 5.

\section{Water Treatment Method}

Water treatment ought to happen before the product conveyed to the user and when it is discharged again. Water sanitization regularly happens on the brink of the last delivery points to lessen pumping prices and the probabilities of the product become adulterated after treatment. Treatment plants for customary surface water usually entails three stages: clarification, distillation and decontamination. Clarification denotes particles 


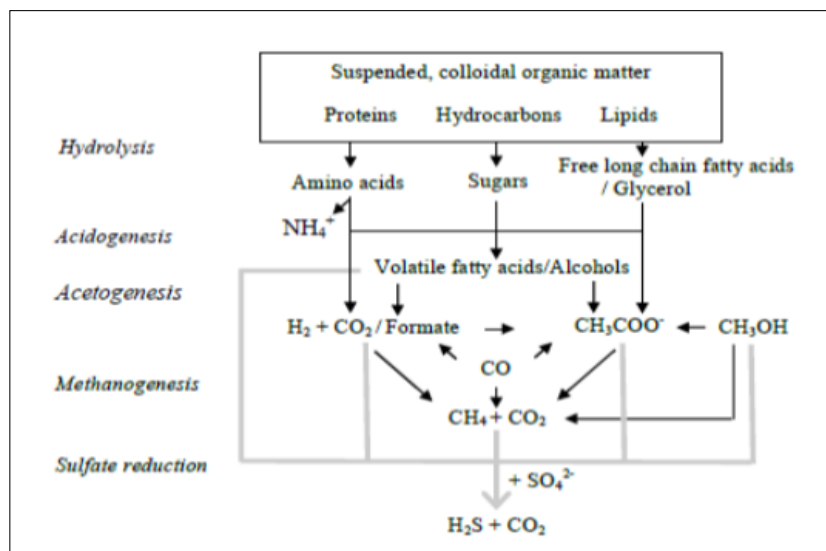

Figure 5: Abridged schematic illustration of the anaerobic degradation method

separation such as filthiness, organic matter etcetera, from the water stream. Chemical addition for instance ferric chloride and alum, disrupts the particle charges besides prepares them for clarification through floating or settling out of the water creek. Actuated carbon filters, sand and anthracite improve the water stream as well remove smaller particle matter although there are other disinfection techniques but chlorine addition is preferable because chlorine efficiently kills most viruses, bacteria as well maintains a residual result to safeguard the water supply from the supply system as shown in Figures $6 a$ and $\mathrm{b}$.

\section{Diverse water test to detect heavy metals, sulphate and bacteria}

Test to detect heavy metals: OES Instrument Archetype can be utilized to measure the heavy metals concentrations, followed by absorption determination and water quality pollution indices can be calculated.

Test for sulfate ions: This can be carried out by using barium chloride with dilute hydrochloric acid and the outcome is a white precipitous if sulphate ions are present as shown in Figure 7.

\section{Physicochemical parameter test}

\section{Bacteriological oxygen demand}

Biochemical or Biological Oxygen Demand (BOD) is the dissolved oxygen quantities required by aerobic organic bacteria to break organic substance available in given water sampling over a specific period of time and at certain temperature. BOD incubators is also called low temperature incubators are one of most essential lab equipment in hospitals, many research centers and other pharmaceutical labs. BOD come in different sizes and capacities from $36 \mathrm{~L}$ to $1000 \mathrm{~L}$, designed to meet APHA specifications and also equipped with mechanical convection system for digital temperature controller, even
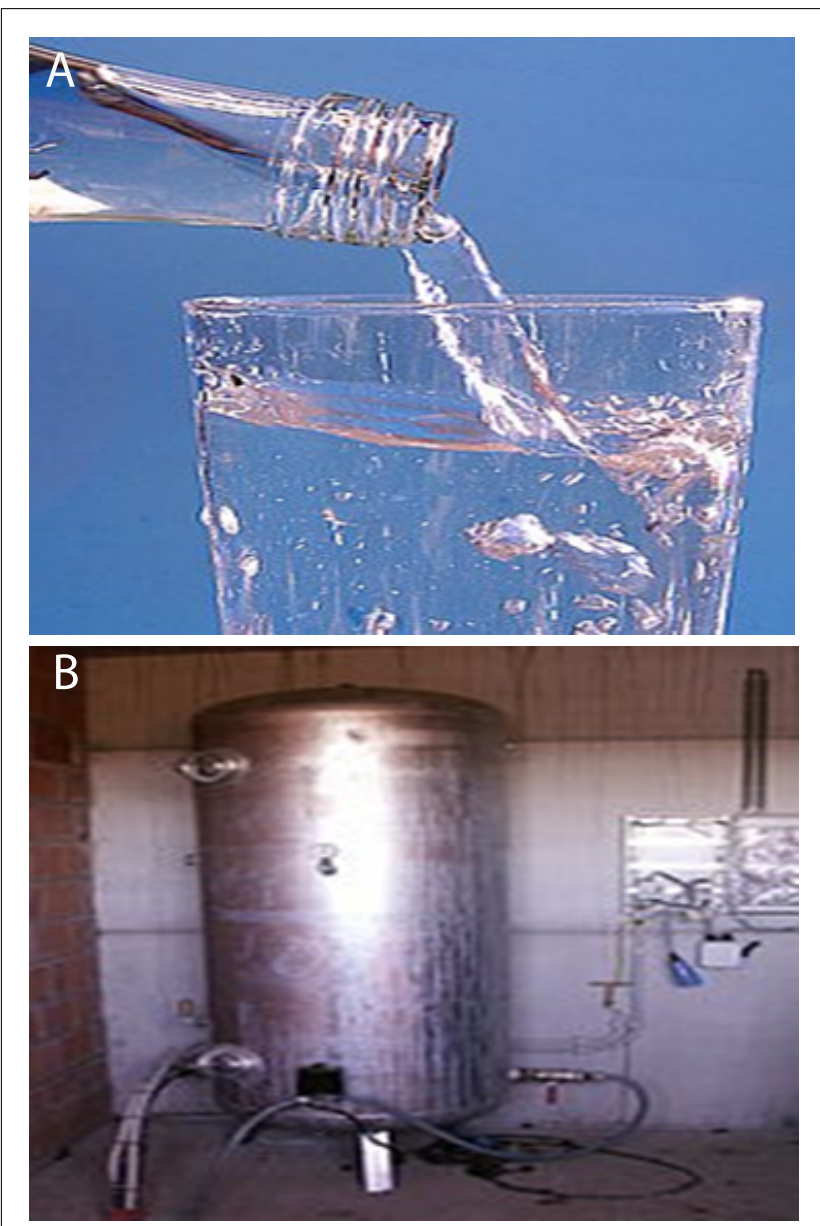

Figure 6: (a) Clean portable water is crucial to human life; (b) Water pressuring needed between the minor water reserve and the consumer

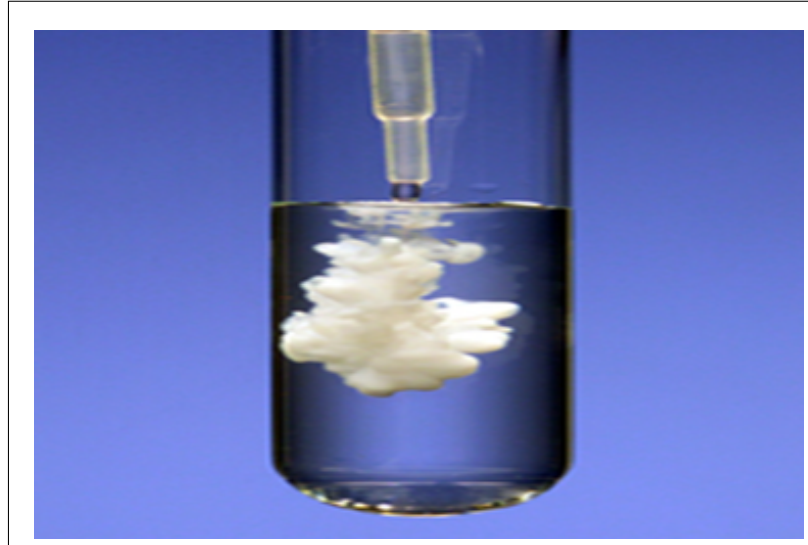

Figure 7: Test for sulfate ions by using barium chloride

air distribution, safety high and low limit controls and overload protection system.

\section{Chemical Oxygen Demand (COD)}

This test can be utilized for ease computation of the organics quantity in water, besides focal implementation 
in measuring corrosion amount available in surface water such as lagoons, streams or wastewater as well useful for water quality through supplies of metric to ascertain the effluent impact on the receiving body. Higher COD levels indicate a bigger quantity of oxidization organic substance in the sampling, which will minimize DO (Dissolved Oxygen) levels. It is usually denoted as mass of oxygen expended over solution volume which in SI units is milligrams per litre $(\mathrm{mg} / \mathrm{L})$ and accepted standard measurement by the International Organization for Standardization is ISO 6060.

\section{Physical test on water}

The physical characteristics of water are paramount as they are the first visibly perceptible factors. Ideally water should be colourless as well odourless with a neutral $\mathrm{pH}$. Presence of dissolved components such as silt, clay, algal materials and so on makes water turbid and change in a $\mathrm{pH}$ make it corrosive.

\section{Conductivity}

Conductivity is the estimation of water's capacity to pass electrical current. Conductive ions obtained from dissolved salts plus inorganic materials like chlorides, sulphides, alkalis and carbonate compounds. More ions generate bigger water conductivity, similarly fewer ions present lesser water conductivity, deionized or sanitized water can act as an insulator since its have very low conductivity value. Conductivity generally measured in micro or milli siemens per centimeter (uS/cm or $\mathrm{mS} / \mathrm{cm}$ ).

\section{pH}

This is the measurement of the relative amount of both free hydrogen and hydroxyl ions present in the water to be precise how acidic or basic water is, ranges from zero to fourteen (0-14), with 7 as neutral. Less than seven (7) $\mathrm{pHs}$ values shows acidity, whereas $\mathrm{pH}$ of greater than seven (7) shows a base. Water with higher free hydrogen ions is acidic, whereas water with lower free hydroxyl ions is basic.

\section{Salinity}

This is the overall absorption of all dissolved salts in water, the electrolytes generate ionic particles as they dissolve and each carries a positive plus negative charge. It can be calculated using a complete chemical analysis but the technique is tough besides time consuming. The units used to measure salinity depends on conductivity values which are unitless, but are regularly followed by the practical salinity units (psu) notation and the salinity oscillate unit based on usage and reporting process that is parts per thousand (ppt) or grams/kilogram (g/kg), also $1 \mathrm{ppt}=1 \mathrm{~g} / \mathrm{kg}$ is used as the standard.

\section{Temperature}

Temperature exerts a vital impact on growth and biological activity also governs the organisms brands that can reside in rivers, lakes etcetera. The chemical reactions rate normally rises at greater temperature.

\section{Total Dissolve Solid (TDS)}

This integrate all ion particles total that are below two microns $(0.0002 \mathrm{~cm})$. Also comprises of all the isolated electrolytes that formed salinity absorptions and other compounds like dissolved biological matter. In clean water, TDS is roughly equal to salinity but in wastewater or contaminated regions, TDS can encompass organic solutes like hydrocarbons, urea and salt ions. This can be reported in $\mathrm{mg} / \mathrm{L}$ as well estimated by calculated by multiplying conductivity value with an empirical factor or gravimetry that is with an evaporation dish.

\section{Remediation Technologies of Heavy Metals}

There are numerous remediation technologies that has been utilized for heavy metals elimination from water or wastewater. Heavy metals bioaccumulation in food chains and their poisonousness to organic systems as a result of concentration increased over time has generated to terrific pressure for their purification and separation (Gionfriddo et al., 2016). Heavy metals can be remove from portable water very easily with consistent technology that are accessible in the market such as: Precipitation plus coagulation, Ion exchange, Membrane clarification, Bioremediation, Heterogeneous photocatalysts (Agnieszka et al., 2018; Moreau, 2017; Fish et al., 2017; Jingqing et al., 2016).

\section{CONCLUSION}

This study examined the impact of sulphate and heavy metals release on household portable water in Nigeria. Though all finished portable water parameters from water distribution system reached water healthy standards, nevertheless the tap water which the users actually exploited can be infested, because the portable water undertake extensive Hydraulic Retaining Period (HRP) of storing and transference in water distribution systems. To achieve success on this subject matter, persistent and periodic awareness programmes through miscellaneous communication mediums is essential so that user will be conscious of portable water contamination. Additionally, consumers must be sensitize of the best way to handle excess sulphate and heavy metals in drinking water; common methods to remove sulphate and heavy metals from portable water; water test to detect presence of heavy metals, sulphate, bacteria and remediation technologies of heavy metals. This study will enlighten the public on importance of having good water with no chemical 
substances that can cause harm to human health through heavy metal contamination as well as guide public water provider on how to mitigate potential release of heavy metals in water supply to the populace, especially water that pass through old pipes. Conclusively, both end user and portable water providers must always observe the portable water from tap, so as to expeditiously detect any potential release of heavy metals due to iron corrosion as it poses contaminants health threat when released into household portable water.

\section{REFERENCES}

Andrade K, Logemann J, Heidelberg KB, Emerson JB, Comolli LR, Hug LA, Probst AJ, Keillar A, Thomas BC, Miller CS, Allen EE, Moreau JW, Brocks JJ and Banfield (2015). Metagenomic and lipid analyses reveal a diel cycle in a hypersaline microbial ecosystem. ISME. J. 9: 2697-2711.

Agnieszka SJ, Zeuschner P, Noga P and Skrzypczak M (2018). Monitoring of heavy metals in selected Water Supply Systems in Poland, in relation to current regulations. Water, Wastewater and Energy in Smart Cities. 1-6.

Balakrishnan A and Ramu A (2016). Evaluation of heavy metal pollution index (HPI) of ground water in and around the coastal area of Gulf of Mannar Biosphere and Palk Strait. J. Adv. Chem. Sci. 2: 331-333.

Brraich S and Jangu S (2015). Evaluation of water quality pollution indices for heavy metal contamination monitoring in the water of Harike wetland (Ramsarsite, Iran). Int. J. Sci. Res. 5: 1-6.

Ediagbonya T, Nmema E, Nwachukwu P and Teniola O (2015). Identification and Quantification of Heavy Metals, Anions and Coliforms in Water bodies using Enrichment Factors. J. Environ. Anal. Chem. 2: 1-9.

Fish K, Osbornc M and Boxall JB (2017). Biofilm structures (EPS and bacterial communities) in drinking water distribution systems are conditioned by hydraulics and influence discoloration. Sci. Total. Environ. 571580 .

Ghaderpoori M, Kamarehie B, Jafari A, Ghaderpoury A and Mohammad Karami A (2018). Heavy metals analysis and quality assessment in drinking water. Data in Brief. 685-692.

\section{Article | Published: 01 August 2016}

Gionfriddo CM, Tate MT, Wick RR, Schultz MB, Zemla A and Michael P (2016). Microbial mercury methylation in Antarctic sea ice. Nat. Microbiol. 1.

Gunatilake S (2015). Methods of Removing Heavy Metals from Industrial Wastewater. J. Multidiscip. Eng. Sci. Stud. 1: 12-18.
Hug K, Maher W, Krikowa F, Foster S and Moreau JW (2017). Experimental evaluation of sampling, storage and analytical protocols for measuring arsenic speciation in sulphidic hot spring waters. Microchem. J. 130: 162167.

Huifang S, Shi B, Yang F and Wang D (2017). Effects of sulfate on heavy metal release from iron corrosion scales in drinking water distribution system. Water. Res. 114: 69-77.

Liu J, Chen H, Yao L, Wei Z, Lou L, Shan Y, Endalkachew SD, Mallikarjuna N, Hu B and Zhou X (2016). The spatial distribution of pollutants in pipe-scale of large-diameter pipelines in a drinking water distribution system. J. Hazard. Mater. 317: 27-35.

Ling YC, Bush R, Grice K, Tulipani S, Berwick L and Moreau JW (2015). Distribution of iron- and sulfurcycling bacteria across a coastal acid sulfate soil (CASS) environment: implications for passive bioremediation by tidal inundation. Front. Microbiol. 6: 624.

Manjie Li, Zhaowei Liu, Yongcan Chen and Yang Hai (2016). Characteristics of iron corrosion scales and water quality variations in drinking water distribution systems of different pipe materials. Water. Research. 106: 593603.

Guiwei L, Yuanxun D, Hongfu X, Junwei J and Shi BY (2018). Characterization and release profile of ( $\mathrm{Mn}, \mathrm{Al})$ bearing deposits in drinking water distribution systems. Chemosphere. 197: 73-80.

Moreau J (2017). Sulfate-reducing bacteria: applications to metals bioremediation and biorecovery. Handbook of Metal-Microbe Interactions and Bioremediation. 623638.

Ab Razak NH, Praveena SM, Aris AZ and Hashim Z (2015). Drinking water studies: A review on heavy metal, application of biomarker and health risk assessment (a special focus in Malaysia). J. Epidemiol. Glob Health. 5: 297-310.

Torres ME, Cox T, Hong WL, McManus J, Sample JC, Destrigneville C, Gan HM, Gan HY and Moreau JW (2015). Crustal Fluids and Ash Alteration Impact on the Biosphere of Shikoku Basin Sediments, Nankai Trough, Japan. Geobiology.11-20.

Li X, Wang H, Hu C, Min Yang, Hu H, Niu J (2015). Characteristics of biofilms and iron corrosion scales with ground and surface waters in drinking water distribution systems Corros. Sci. 90: 331-339.

Watts MP, Gan HM, Peng LY, Lê Cao KA and Moreau JW (2017). In situ stimulation of thiocyanate biodegradation through nutrient amendment in gold mine tailings water. Environ. Sci. Technol. 51: 13353-13362. 